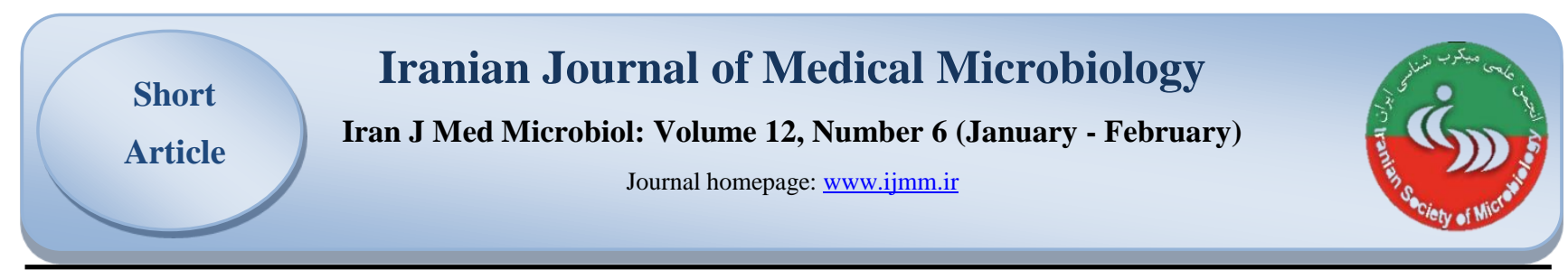

\title{
An Epidemiological Study on the Status of Hydatid cyst in Surgical Patients in Golestan Hospital of Ahwaz During 2002-2011
}

\author{
Jasem Saki $^{1,2}$, Iman khodkar $^{1,2}$, Leila Hardani Pasand ${ }^{1,2^{*}}$, Iraj Nazari ${ }^{3}$ \\ 1.Department of Medical Parasitology,School of Medicine, Ahvaz Jundishapur University of Medical Sciences, Ahvaz, Iran \\ 2.Department of Surgery, Golestan Hospital, Ahvaz Jundishapur University of Medical Sciences, Ahvaz, Iran \\ 3.Student Research Committee, Ahvaz, Jundishapur University of Medical Sciences, Ahvaz, Iran
}

\section{Article Information}

\section{Article history:}

Received: 2018/02/10

Accepted: 2019/01/07

Available online: 2019/03/06

Article Subject:

Medical Parasitology

IJMM 2019; 12(6): 442-446

Corresponding author:

Leila Hardani Pasand

Department of Medical parasitology,School of

Medicine, Ahvaz Jundishapur

University of Medical

Sciences, Ahvaz, Iran

Email:

hardani2013@gmail.com

Use your device to scan

and read the article online

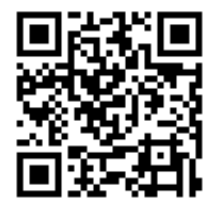

\section{Abstract}

Background and Aims: Hydatidosis is one of the most important zoonotic parasitic diseases. Hydatidosis is endemic in Iran and is the cause of hospitalization of almost $1 \%$ of patients in surgical wards. The purpose of this study is to examine the epidemiologic status of hydatid cyst in patients undergoing surgery in Golestan hospital of Ahvaz during 2002-2011 using archived files of the patients.

Materials and Methods: This research is a cross-sectional study. During the mentioned period, 2002 until 2011, 55 patients in Ahvaz Golestan hospital have undergone hydatid cyst surgery and the information in the patients' files were examined by referring to the relevant archives in the mentioned hospital.

Results \& Conclusion: Among the 55 patients studied, 37 (67.3\%) were female and 18 patients $(32.7 \%)$ were male. The highest incidence rate was found in liver with 47 cases $(85.5 \%)$, followed by lung with 5 cases $(9 \%)$. Considering the results, the highest prevalence rate was found among urban residents $(\mathrm{n}=33,60 \%)$ whilst 22 cases $(40 \%)$ belonged to the rural residents. The results of this study indicate that the occurrence of the disease was significant in Khuzestan province during the mentioned period which reflects the necessity of more comprehensive and updated studies.

How to cite this article:

Hardani Pasand L, Saki J, Khodkar I, Nazari I. An Epidemiological Study on the Status of Hydatid cyst in Surgical Patients in Golestan Hospital of Ahwaz During 2002-2011. Iran J Med Microbiol. 2019; $12(6): 442-446$ 


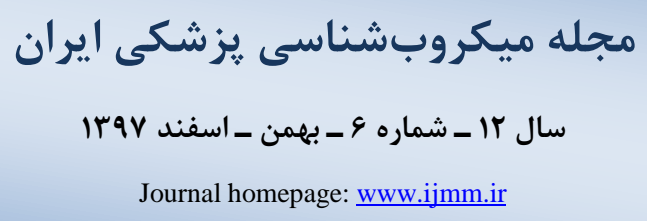

Journal homepage: www.ijmm.ir

\section{وضعيت إيدميولوزيك كيست هيداتيك در بيماران جراحى شده در بيمارستان كلستان

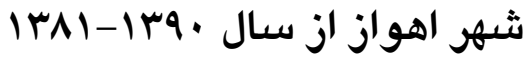

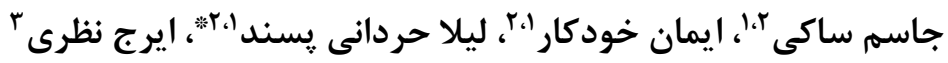

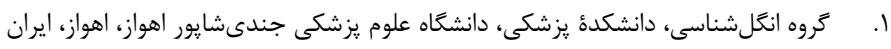

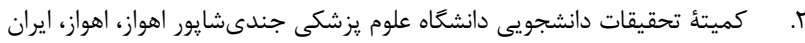

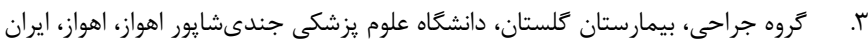

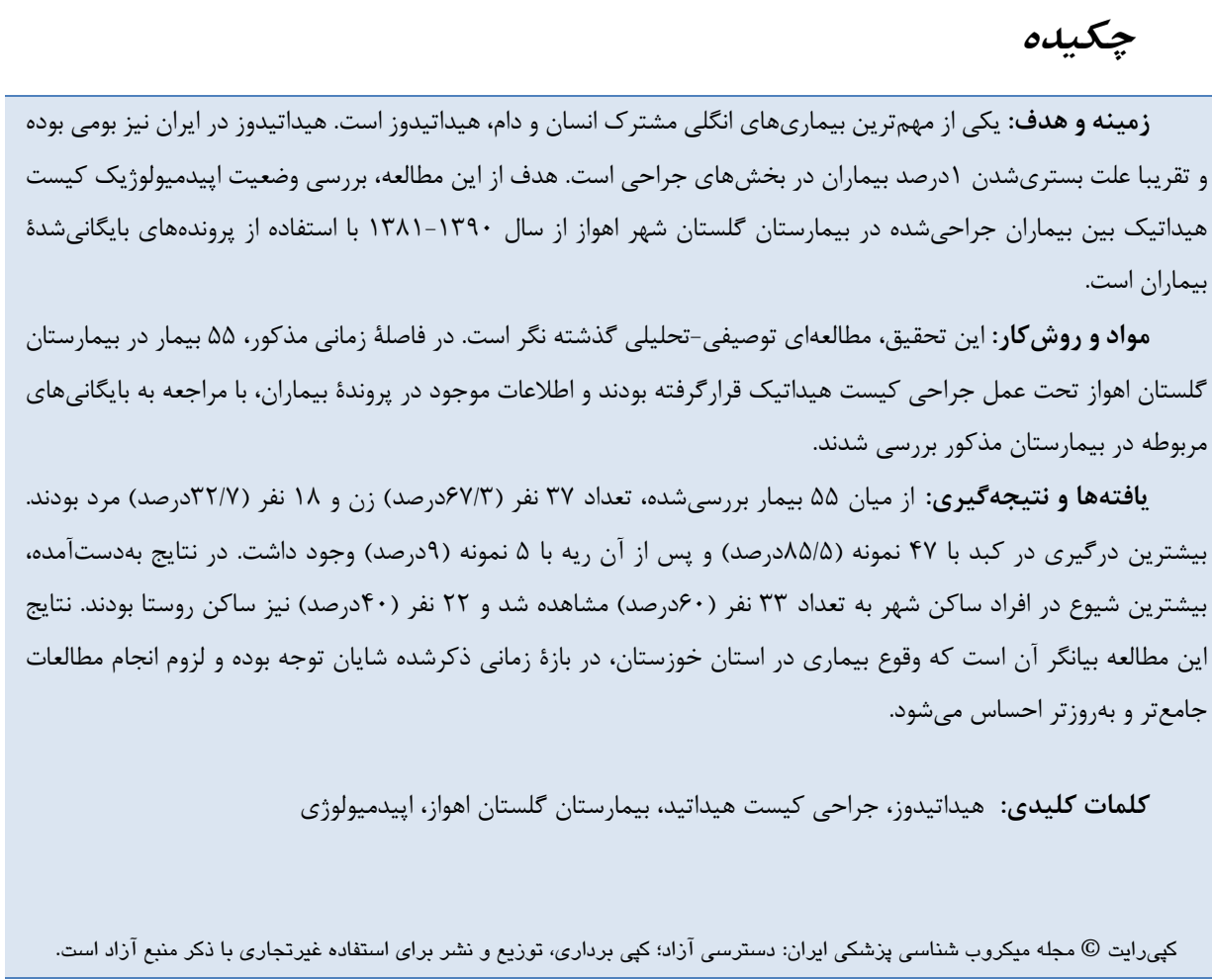

اطلاعات مقاله

زمينه و هدف: يكى از مههمترين بيمارى هاى انتلى مشترى انسان و دام، هيداتيدوز است. هيداتيدوز در ايران نيز بومى بوده

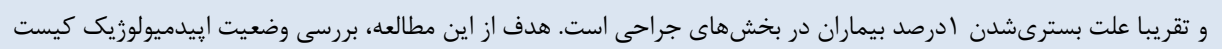

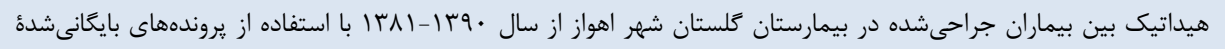
بيماران است مواد و روش كار: اين تحقيق، مطالعهاى توصيفى-تحليلى كذشته نكَر است. در فاصله زمانى مذكور، لها بيمار در بيمارستان مربوطه در بيمارستان مذكور بررسى شدند. 


\section{بافنتها و بحث}

اكينوكوكوزيس در انسان به دو شكل تكحفرهاى و

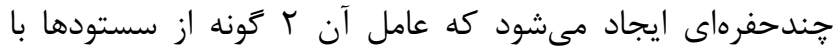
نامهاى اكينوكوكوس كرانولوزوس و اكينوكوكوس مولتى لكولاريس هستند. در ايران شكل تك حفرهاى بيمارى ايجادشده بلوسيلة

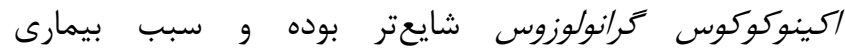
هيداتيدوزيس مىشود كه از لحاظ بهداشتى و اقتصادى مشكلات فراوانى را جه براى فرد مبتلا و جهه براى سيستم بهود بهداشتى جامعه

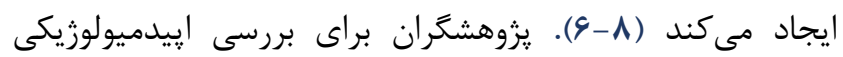

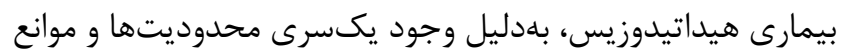

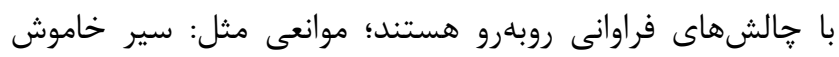

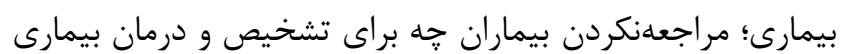

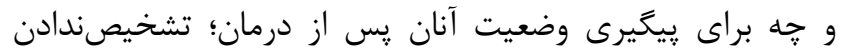

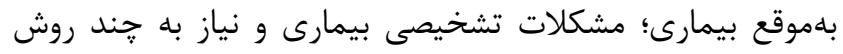

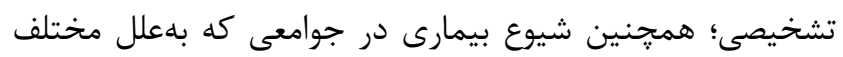

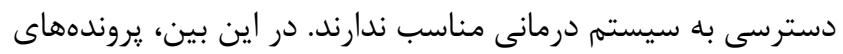

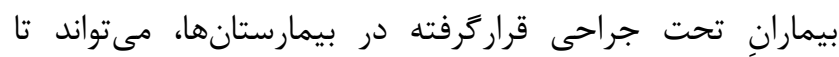

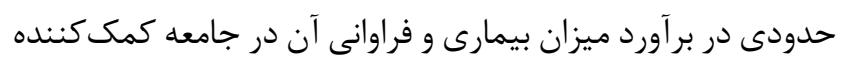

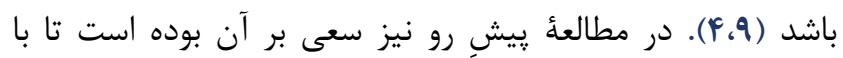

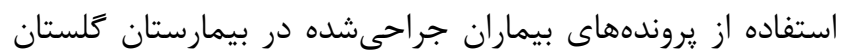

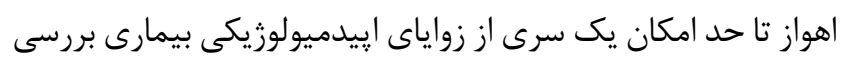
شود.

در مطالعُٔ حاضر، از ميان فه بيمار بررسىشده، تعداد

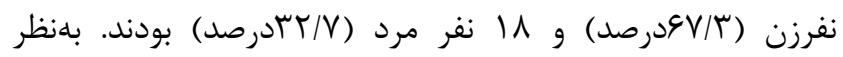
مىرسد مواجههُ زنان، بهويزه زنان خانهدار با عامل بيمارى، بهعلت نقش اصلى آنها در تهيه و طبخ موادغذايى خانواده، در مقايسه با بها

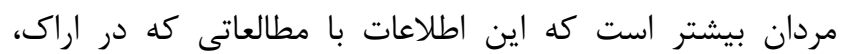
زنجان،ياسوج و مطالعهاى كه به مرور سيستماتيك وضعيت كيست

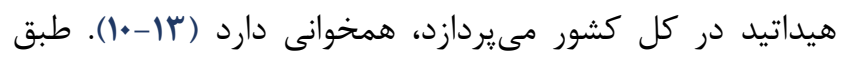
مطالعات انجامشده نوزاد كرم ، اكينوكوكوس كرانولوزوس در ميزبان واسط قادر به ايجاد كيست مى باشد و شايع ترين اندام تشكيل كيست در ميزبان واسط كبد است (q،q). در مطالعهُ حاضر نيز

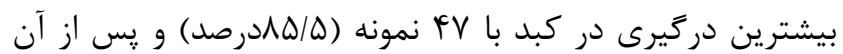

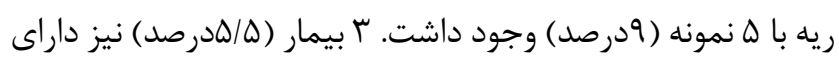
درگيرى در هر دو اركان كبد و ريه بودند (نمودار شماره () ).
(ץ). هيداتيدوز در كشور ما نيز بومى بوده و طبق مطالعات انجامشده، مناطق جنوبى كشور، داراى بيشترين ميزان شيوع اين

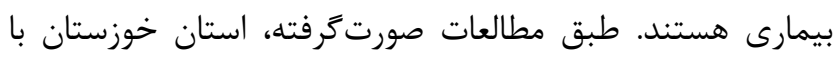
شيوع سرمى I/VA ادرصد، بيشترين ميزان شيوع بيمارى هيداتيدوز

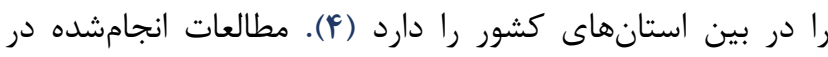
خصوص اييدميولوزى هيداتيدوز در نقاط مختلف كشور، حاكى از شيوع نسبتا وسيع اين بيمارى در نقاط مختلف كشور است؛ بهعنوان

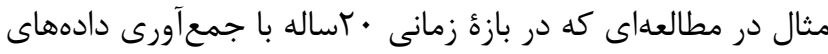
بيمارستانى در شهر مشهد صورت گرفت، نشان داده شد كه فراوانى

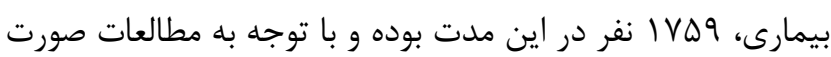
كرفته، ميزان فراوانى بيمارى در شرق كشور بسيار زياد است (ه).

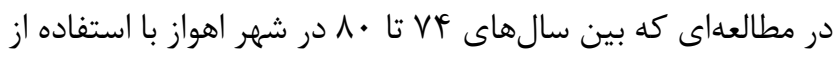
يروندهاى بيمارستانى انجام كرفت، تعداد ·f أمونه بيمارى اعلام

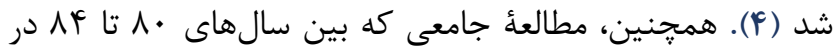
سطح كشور صورت گرفت، نشان داد كه شيوع كلى اين بيمارى در

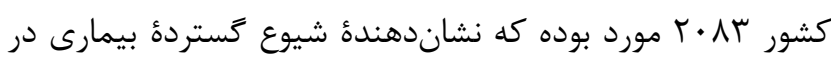
مناطق مختلف كشور است (f). براى بررسى إيدميولوزى هيداتيدوز، با توجه به شيوع جشمگير اين بيمارى، دادههاى

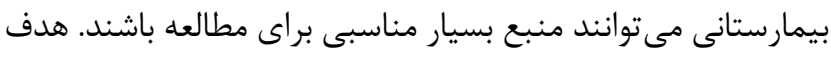

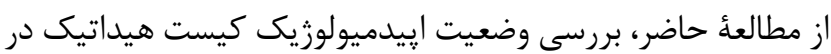
بيماران جراحىشده در بيمارستان كلستان شهر اهواز، بهعنوان يكى

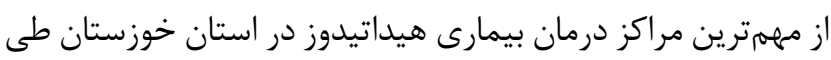

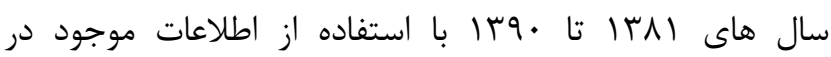
يروندهاى بايعانى شده بيماران مبتلا است.

\section{مواد و روش ها}

تحقيق ييشي رو، از نوع مطالعات توصيفى -تحليلى گذشته نخر

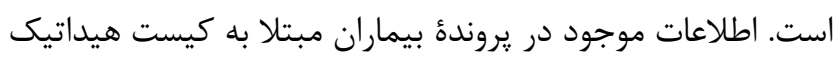

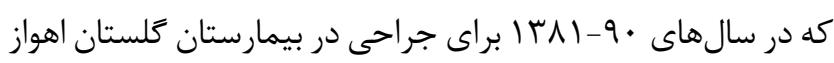

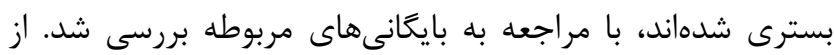

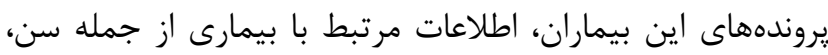

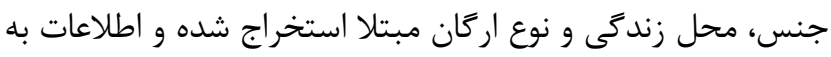

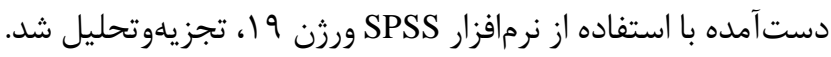
همجنين بهمنظور تهيةُ نمودارهاى فراوانى، روش آمار توصيفى به

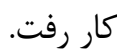


مطالعات إيدميولوزيك مى توانند جزئيات بسيار دقيق و مبسوطى را از وضعيت كذشته و فعلى بيمارى در اختيار متوليان

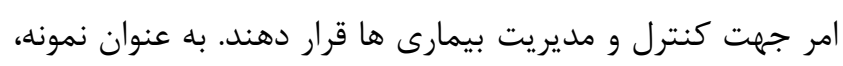

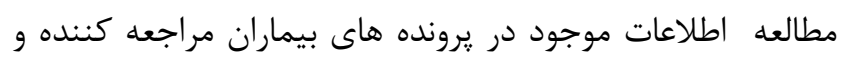

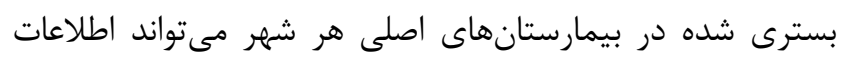

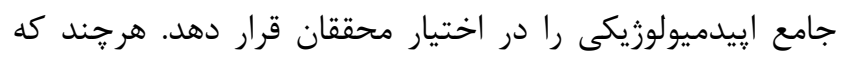

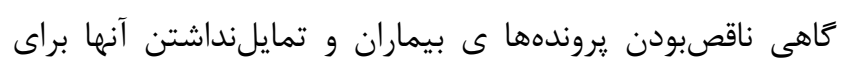

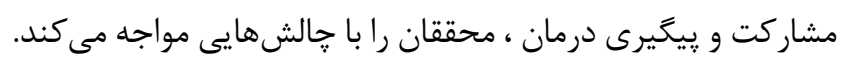

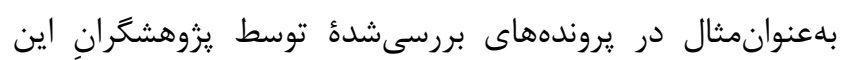

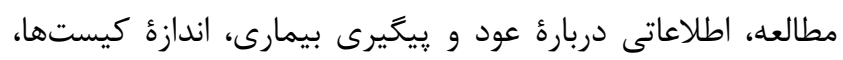

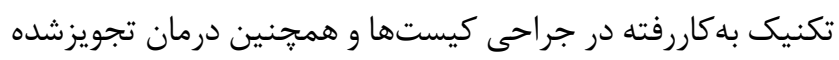
يس از جراحى يافت نشد. در كشور ما مطالعات بسيارى در زمينه

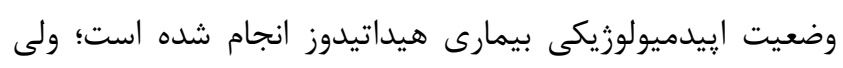

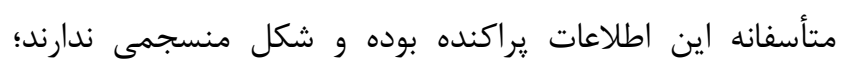

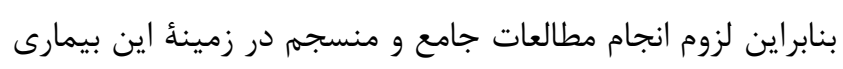

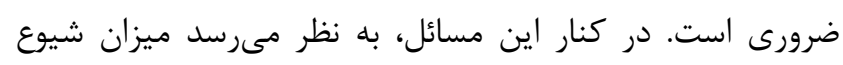

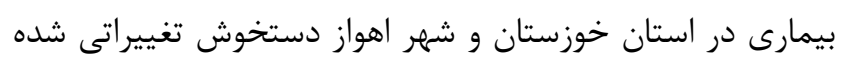

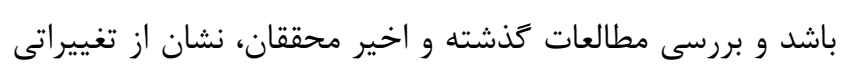
در ميزان شيوع بيمارى در استان خوزستان و مركز آن شهر آهواز دارد و بررسى هاى بيشتر و جامعترى در اين زمينه لازم است (19،

نتايج اين مطالعه بيانكَ آن است كه وقوع بيمارى در شهر

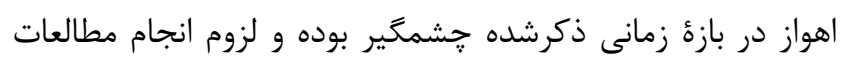

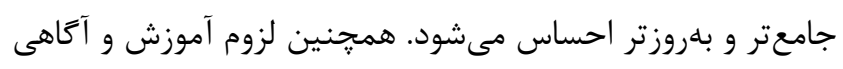

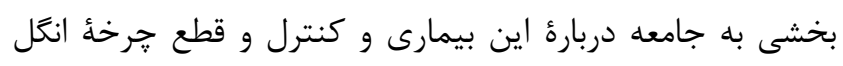

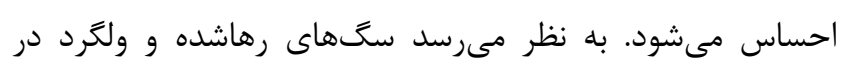

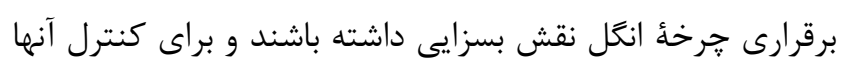

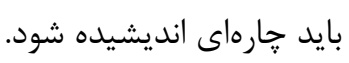

\section{سياسخَزارى}

بدينوسيله از جناب آقاى دكتر ييمان زارع، آقاى دكتر

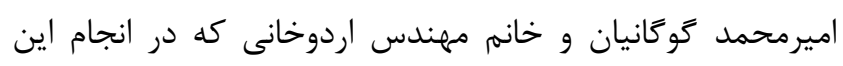

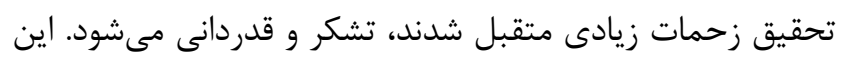

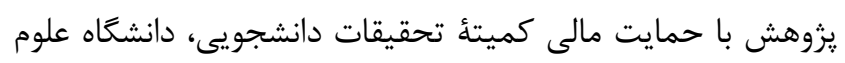

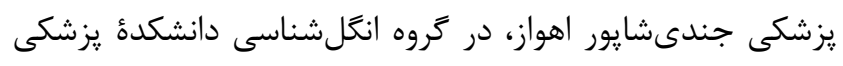

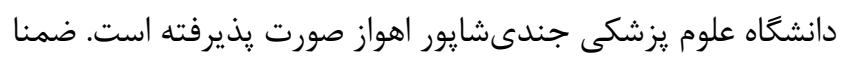

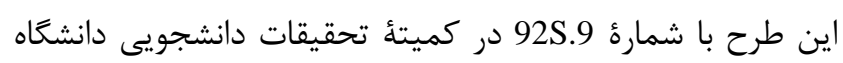
علوم يزشكى جندى شايور اهواز به ثبت رسيده است.

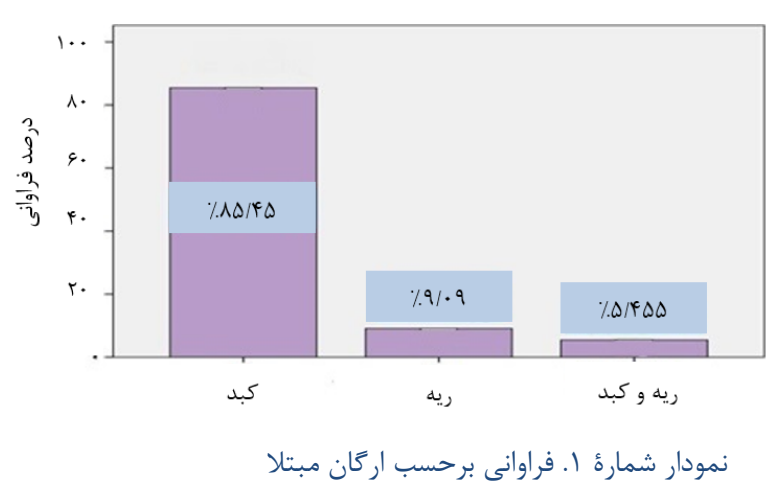

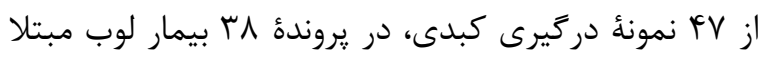

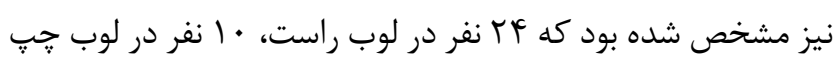

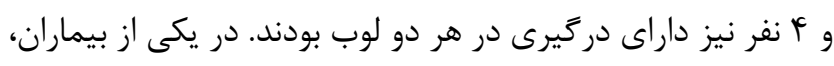

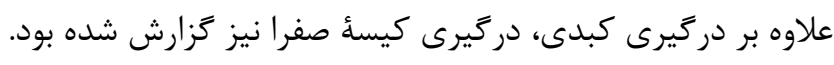

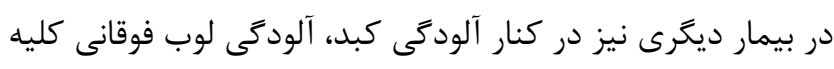

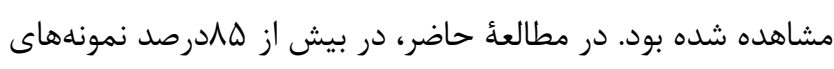

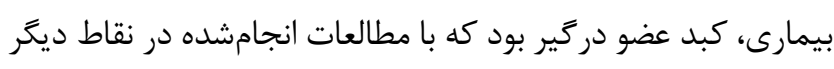

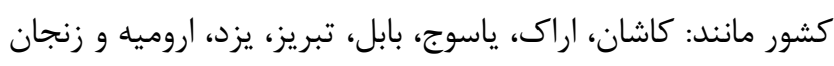

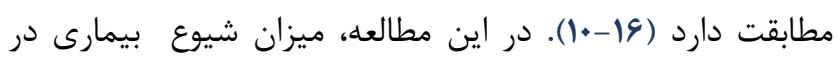

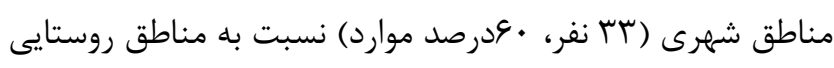

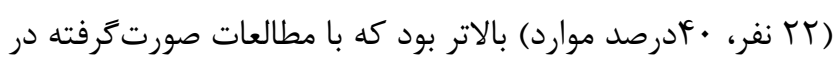

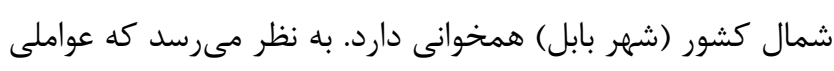

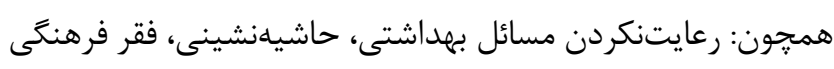

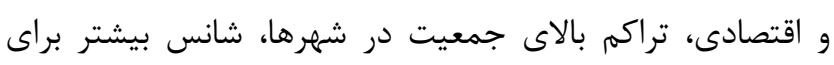

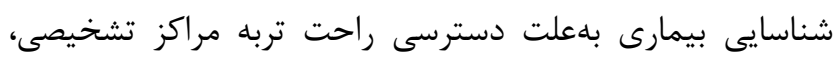

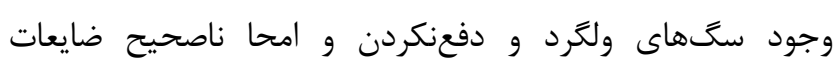

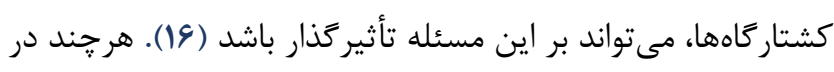

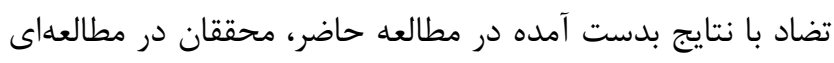

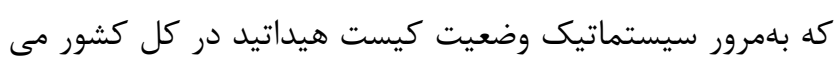

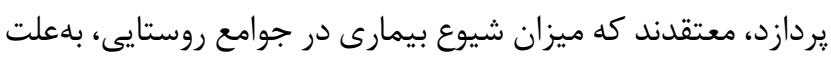

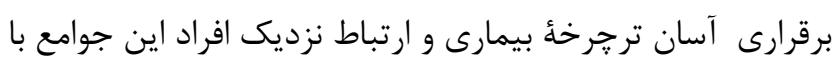

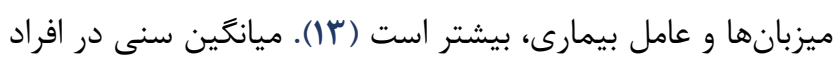

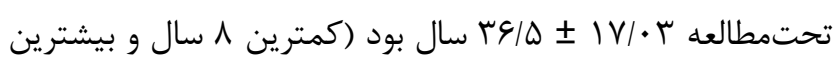

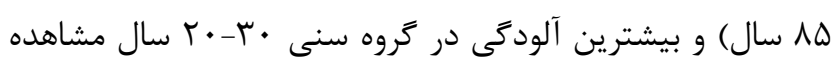

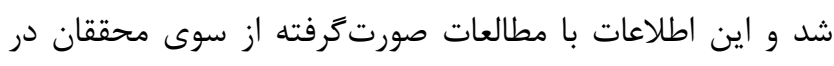

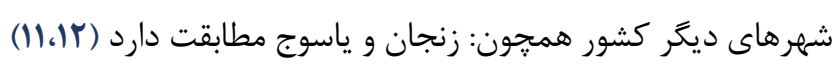

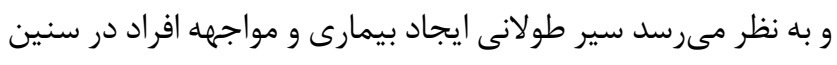
يايين با عامل بيمارى، دركيرى بيشتر بيمارى را در اين مطالعه توجيه مى نمايد. 


$$
\text { بين نويسند }
$$

\section{References}

1. Dalimi A, Motamedi GH, Hosseini M, Mohammadian B, Malaki H, Ghamari Z, Far FG. Echinococcosis/hydatidosis in western Iran. Vet Parasitol. 2002; 105(2): 161-71. https://doi.org/10.1016/S0304-4017(02)00005-5

2. Dakkak A. Echinococcosis/hydatidosis: a severe threat in Mediterranean countries. Veterinary Parasitology. 2010; 174(1-2): 2-11. https://doi.org/10.1016/j.vetpar.2010.08.009

3. Dziri C, Haouet K, Fingerhut A. Treatment of hydatid cyst of the liver: where is the evidence?. World journal of surgery. 2004; 28(8): 731-6. https://doi.org/10.1007/s00268-004-7516-z

4. Gholami S, Tanzifi A, Sharif M, Daryani A, Rahimi MT, Mirshafiee S, Sarvi S. Demographic aspects of human hydatidosis in Iranian general population based on serology: A systematic review and meta-analysis. Veterinary world. 2018; 11(10): 1385. https://doi.org/10.14202/vetworld.2018.1385-1396

5. Amouian S, Tayebi N, Mohamadian Roshan N. A retrospective study of 1759 cases of Hydatid cyst in Mashad University hospitals. Hakim Research Journal. 2005; 4(7): 7-13.

6. Budke CM, Deplazes P, Torgerson PR. Global socioeconomic impact of cystic echinococcosis. Emerging infectious diseases. 2006; 12(2): 296. https://doi.org/10.3201/eid1202.050499

7. Craig PS, McManus DP, Lightowlers MW, Chabalgoity JA, Garcia HH, Gavidia CM, et al. Prevention and control of cystic echinococcosis. The Lancet infectious diseases. 2007; 7(6): 385-94. https://doi.org/10.1016/S1473-3099(07)70134-2

8. Harandi MF, Budke CM, Rostami S. The monetary burden of cystic echinococcosis in Iran. PLOS neglected tropical diseases. 2012; 6(11): e1915. https://doi.org/10.1371/journal.pntd.0001915

9. Smego RA, Sebanego P. Treatment options for hepatic cystic echinococcosis. J Infect Dis. 2005; 9(2): 69-76. https://doi.org/10.1016/j.ijid.2004.08.001

10. Davami M. Evaluation of operated patients with hydatid cyct in Markazi province. Journal of Arak University of Medical Sciences. 1994; 2(5): 12-15.
11. Nurian A, Zargham D, Nourizadeh H. Evaluation of operated cases of hydatid cyct in Shafieeye hospital during 1984-1994. Journal of Zanjan University of Medical Sciences and Health Services. 1993; 16(4): 22-8.

12. Sarkari B, Sadjjadi SM, Beheshtian MM, Aghaee M, Sedaghat F. Human cystic Echinococcosis in Yasuj district in Southwest of Iran: an epidemiological study of seroprevalence and surgical cases over a ten-year period. Zoonoses and public health. 2010; 57(2): 146-50.

13. Khalkhali HR, Foroutan M, Khademvatan S, Majidiani H, Aryamand S, Khezri P, Aminpour A. Prevalence of cystic echinococcosis in Iran: a systematic review and meta-analysis. Journal of helminthology. 2018; 92(3): 260-8. https://doi.org/10.1017/S0022149X17000463

14. Arbabi MO, Hooshyar H. Survey of echinococcosis and hydatidosis in Kashan region, central Iran. Iranian Journal of Public Health. 2006; 35(1): 75-81.

15. Shir Yazdi SM, Mir Shamsi MH, Hosseini B, Ebadi M. Cases of the Hydatid cyst that were operated upon in Yazd. Journal of Shahed Sadoughi University of Medical Scienced and Health and Health Services. 2000; 1(8): 2530 .

16. Ghaffari S. Study of operated hydatid cysts cases in three medical centers of Babol Medical University during 1991-96. Journal of babol University of Medical Sciences. 1999; 1(1): 27-33.

17. Nourjah N, Sahba G, Baniardalani M, Chavshin A. Study of 4850 operated hydatidosis cases in Iran. Southeast Asian J Trop Med Public Health. 2004; 35(1): 218-22.

18. Rafiei A, Hemadi A, Maraghi SK, Craig PS. Human cystic echinococcosis in nomads of south-west Islamic Republic of Iran. Eastern Mediterranean Health Journal. 2007; 13(1): 41-9.

19. Kasaei R, Tavalla M, Etebar H. Serological survey of Echinococcus granulosus in nomads of southwest Iran using the ELISA method during 2014-15. Infez Med. 2016; 24(1): 43-7. 